\title{
Unraveling the acoustic electron-phonon interaction in graphene
}

\author{
Kaasbjerg, Kristen; Thygesen, Kristian S.; Jacobsen, Karsten W.
}

Published in:

Physical Review B Condensed Matter

Link to article, DOI:

10.1103/PhysRevB.85.165440

Publication date:

2012

Document Version

Publisher's PDF, also known as Version of record

Link back to DTU Orbit

Citation (APA):

Kaasbjerg, K., Thygesen, K. S., \& Jacobsen, K. W. (2012). Unraveling the acoustic electron-phonon interaction in graphene. Physical Review B Condensed Matter, 85(16), 165440.

https://doi.org/10.1103/PhysRevB.85.165440

\section{General rights}

Copyright and moral rights for the publications made accessible in the public portal are retained by the authors and/or other copyright owners and it is a condition of accessing publications that users recognise and abide by the legal requirements associated with these rights.

- Users may download and print one copy of any publication from the public portal for the purpose of private study or research.

- You may not further distribute the material or use it for any profit-making activity or commercial gain

- You may freely distribute the URL identifying the publication in the public portal

If you believe that this document breaches copyright please contact us providing details, and we will remove access to the work immediately and investigate your claim. 


\title{
Unraveling the acoustic electron-phonon interaction in graphene
}

\author{
Kristen Kaasbjerg, ${ }^{*}$ Kristian S. Thygesen, and Karsten W. Jacobsen \\ Center for Atomic-scale Materials Design (CAMD), Department of Physics, \\ Technical University of Denmark, DK-2800 Lyngby, Denmark
}

(Received 12 January 2012; published 23 April 2012)

\begin{abstract}
Using a first-principles approach we calculate the electron-phonon couplings in graphene for the transverse and longitudinal acoustic phonons. Analytic forms of the coupling matrix elements valid in the long-wavelength limit are found to give an almost quantitative description of the first-principles matrix elements even at shorter wavelengths. Using the analytic forms of the coupling matrix elements, we study the acoustic phonon-limited carrier mobility and quasiparticle lifetime observable in photoemission spectroscopy for temperatures 0-200 K and high carrier densities of $10^{12}-10^{13} \mathrm{~cm}^{-2}$. We find that the intrinsic effective acoustic deformation potential of graphene is $\Xi_{\text {eff }}=6.8 \mathrm{eV}$ and that the temperature dependence of the mobility $\mu \sim T^{-\alpha}$ in the Bloch-Grüneisen regime increases beyond an $\alpha=4$ dependence even in the absence of screening when the true coupling matrix elements are considered. The $\alpha>4$ temperature dependence of the mobility is found to originate in a similar temperature dependence of the relaxation time at the Fermi level. The large disagreement between our calculated deformation potential and those extracted from experimental measurements $(18-29 \mathrm{eV})$ indicates that additional or modified acoustic phonon-scattering mechanisms are at play in experimental situations.
\end{abstract}

DOI: 10.1103/PhysRevB.85.165440

PACS number(s): 72.80.Vp, 72.10.-d, 73.40.-c

\section{INTRODUCTION}

Since the experimental realization of graphene, ${ }^{1}$ its electronic properties and their understanding have been studied extensively both experimentally and theoretically. ${ }^{2-4}$ While the intrinsic carrier mobility of graphene is predicted to be exceptionally high, the experimental reality in substrate supported graphene involving charged impurities, electron-hole puddles, surface-optical phonons of the substrate, and disorder typically results in strongly reduced mobilities compared to the expected intrinsic value. ${ }^{5,6}$ Together with scattering on acoustic phonons which manifests itself in a linear temperature dependence of the mobility at higher temperatures, these extrinsic scattering mechanisms typically dominate the mobility in graphene samples.

The linear temperature dependence characteristic for acoustic phonon scattering has so far been observed in both supported $^{5,7-10}$ and suspended ${ }^{11}$ graphene samples. With the recent improvements in sample fabrication, the relative role of acoustic phonon scattering must be expected to become increasingly important in future devices. For example, samples with the commonly used $\mathrm{SiO}_{2}$ substrate replaced by hexagonal boron nitride (h-BN) which has a lattice constant very close to that of graphene and an almost atomically flat surface with strongly reduced disorder, ${ }^{12,13}$ have shown highly improved transport characteristics with mobilities approaching that of suspended graphene. ${ }^{8,14,15}$ Furthermore, the high energy of the surface-optical phonons of h-BN results in a significant reduction of surface-optical phonon scattering ${ }^{16-18}$ that for commonly used gate oxides starts to dominate the mobility around $T \sim 150-200 \mathrm{~K}^{5,7}$

When the mobility is dominated by acoustic phonon scattering, two transport regimes separated by the Bloch-Grüneisen (BG) temperature $T_{\mathrm{BG}}=2 \hbar k_{F} c_{\mathrm{ph}} / k_{\mathrm{B}}$ can be identified. ${ }^{19}$ Here, $k_{F}$ is the Fermi wave vector, $c_{\mathrm{ph}}$ the sound velocity, and $k_{\mathrm{B}}$ the Boltzmann constant $\left(T_{\mathrm{BG}} \sim 57 \mathrm{~K} \sqrt{n}\right.$ for the longitudinal acoustic phonon with the two-dimensional carrier density $n$ measured in units of $10^{12} \mathrm{~cm}^{-2}$ ). Below the BG temperature which corresponds to the acoustic phonon energy $\hbar \omega_{\mathbf{q}}=\hbar c_{\mathrm{ph}} q$ for full backscattering at the Fermi level, short-wavelength acoustic phonons are frozen out restricting scattering processes to small scattering angles. The restricted phase space available for phonon scattering at $T<T_{\mathrm{BG}}$ results in a transition from the linear $\rho \sim T$ high-temperature behavior of the resistivity to a stronger $\rho \sim T^{\alpha}$ temperature dependence in the BG regime where $\alpha=4(\alpha=6)$ in the absence (presence) of screening by the carriers themselves. ${ }^{20,21}$ The BG behavior in the temperature dependence of the mobility (resistivity) has recently been observed experimentally. ${ }^{9}$

As the resistivity is intimately connected to the lifetime of the electronic quasiparticles at the Fermi level, a similar $\mathrm{BG}$ behavior in the temperature dependence of the quasiparticle lifetime is expected. The lifetime of the electronic carriers in graphene can be probed with, e.g., angle-resolved photoemission spectroscopy (ARPES) which provides detailed information about the dominating decay mechanisms of quasiparticles. ${ }^{22-24}$ Unlike transport measurements, this technique can probe quasiparticles at energies far away from the Fermi level. In order to explain experimental ARPES spectra of graphene, theoretical works have focused on the role of optical phonons and electron-electron interactions. ${ }^{25-29}$ However, as demonstrated by transport measurements, there exist an energy window centered around the Fermi level where acoustic phonon scattering may dominate, and hence where $\mathrm{BG}$ behavior in the quasiparticle lifetime should be observable. Indeed, ARPES is often carried out at low temperatures and high carrier densities where BG physics appears. ${ }^{22,23}$

Existing theoretical ${ }^{20,21}$ and experimental ${ }^{5,7-9,11}$ studies of acoustic phonon-limited transport in graphene often parametrize the interaction with acoustic phonons in terms of a coupling to a single effective acoustic phonon. The associated deformation potential coupling constant is extracted from the experimentally measured temperature dependence of the resistivity and ranges from $\sim 18$ to $29 \mathrm{eV}^{5,7-9,11}$ On the other hand, theoretical studies of the acoustic electronphonon coupling yield much lower values on the order of 3.9-7.4 eV. ${ }^{30-32}$ At the same time, different forms of the 
coupling matrix element are used in theoretical studies ${ }^{20,32}$ making a direct comparison of the different values of the deformation potential difficult.

Even though the effect of acoustic phonon scattering on the transport properties of graphene has been studied widely in the literature, ${ }^{20,30,31,33-37}$ a complete study considering the full details of the coupling matrix element is still lacking. The purpose of the present study is to fill out this gap and provide a detailed analysis of the acoustic electron-phonon interaction in graphene and at the same time establish the intrinsic value of the effective deformation potential. We shall focus on supported graphene where flexural phonons are quenched and hence include only the transverse (TA) and longitudinal (LA) acoustic phonons. We use a first-principles method to calculate the electron-phonon interaction ${ }^{38,39}$ supported by the grouptheoretical considerations of Ref. 40 to derive analytic forms of the coupling matrix elements. We then study the intrinsic phonon-limited mobility using a Boltzmann equation approach and the quasiparticle lifetime in the temperature regime 0$200 \mathrm{~K}$ and for high carrier densities $n \sim 10^{12}-10^{13} \mathrm{~cm}^{-2}$, where screening by the carriers suppresses other scattering mechanisms and BG physics appear. We shall restrict the discussion to $n$-doped graphene where the Fermi level resides in the upper Dirac cone and is given by $\varepsilon_{F} \sim 11.65 \sqrt{n} \mathrm{meV}$ with the carrier density $n$ measured in units of $10^{10} \mathrm{~cm}^{-2}$.

\section{THEORY}

In the following, the carriers in graphene are described by massless Dirac fermions with linear dispersion $\varepsilon_{\mathbf{k}}=$ $\hbar v_{F} k$, where $v_{F} \sim 1.0 \times 10^{6} \mathrm{~m} / \mathrm{s}$ is the Fermi velocity. The electronic wave functions are given by $\chi_{\mathbf{k}}(\mathbf{r})=1 / \sqrt{A} e^{i \mathbf{k} \cdot \mathbf{r}} \chi_{\mathbf{k}}$, where $\chi_{\mathbf{k}}=1 / \sqrt{2}\left(\exp \left[-i \theta_{\mathbf{k}} / 2\right], \exp \left[i \theta_{\mathbf{k}} / 2\right]\right)$ is the twocomponent spinor of the atomic $A, B$ sublattices of graphene and $\theta_{\mathbf{k}}$ is the angle of wave vector $\mathbf{k}$.

Within the Boltzmann equation approach, ${ }^{6,34,41}$ the mobility in graphene in the presence of (quasi)elastic scattering mechanisms is given by

$$
\mu_{x x}=\frac{\sigma_{x x}}{n e}=\frac{e v_{F}^{2}\left\langle\tau_{\mathbf{k}}\right\rangle}{2},
$$

where $\sigma_{x x}$ is the conductivity and the density-of-states averaged relaxation time (in units of time per energy) is defined by

$$
\left\langle\tau_{\mathbf{k}}\right\rangle=\frac{1}{n} \int d \varepsilon_{\mathbf{k}} \rho\left(\varepsilon_{\mathbf{k}}\right)\left(-\frac{\partial f}{\partial \varepsilon_{\mathbf{k}}}\right) \tau_{\mathbf{k}} .
$$

Here, $\rho\left(\varepsilon_{\mathbf{k}}\right)=\left(g_{s} g_{v} / 2 \pi \hbar^{2}\right) \varepsilon_{\mathbf{k}} / v_{F}^{2}$ is the density of states of the graphene layer and $g_{s}=2$ and $g_{v}=2$ are the spin and valley degeneracies, respectively. At low temperatures and high carrier densities, where $\varepsilon_{F} \gg k_{\mathrm{B}} T$, this yields $\mu_{x x} \approx$ $e v_{F}^{2} \tau_{k_{F}} / \varepsilon_{F}$.

In the case of acoustic phonon scattering which can be treated as a quasielastic process, the relaxation time for each of the acoustic phonons is given by ${ }^{20}$

$$
\frac{1}{\tau_{\mathbf{k} \lambda}}=\sum_{\mathbf{k}^{\prime}}\left(1-\cos \theta_{\mathbf{k}, \mathbf{k}^{\prime}}\right) P_{\mathbf{k k}^{\prime}}^{\lambda} \frac{1-f_{\mathbf{k}^{\prime}}}{1-f_{\mathbf{k}}},
$$

where $\lambda$ denotes the acoustic phonon branch, $\theta_{\mathbf{k}, \mathbf{k}^{\prime}}$ is the scattering angle and $f_{\mathbf{k}}=f\left(\varepsilon_{\mathbf{k}}\right)$ the Fermi function. The
TABLE I. Material parameters for graphene used in the present work. The phonon related parameters have been obtained from first principles as described in the text. The calculated sound velocities are in excellent agreement with the values reported in Ref. 35.

\begin{tabular}{lcc}
\hline \hline Parameter & Symbol & Value \\
\hline Lattice constant & $a$ & $2.46 \AA(\mathrm{LDA})$ \\
Ion mass density & $\rho$ & $7.6 \times 10^{-8} \mathrm{~g} / \mathrm{cm}^{2}$ \\
Fermi velocity & $v_{F}$ & $1.0 \times 10^{6} \mathrm{~m} / \mathrm{s}$ \\
Transverse sound velocity & $c_{\mathrm{TA}}$ & $14.1 \times 10^{3} \mathrm{~m} / \mathrm{s}$ \\
Longitudinal sound velocity & $c_{\mathrm{LA}}$ & $21.2 \times 10^{3} \mathrm{~m} / \mathrm{s}$ \\
Electron-phonon couplings & & \\
Transverse & $\beta_{\mathrm{TA}}$ & $2.8 \mathrm{eV}$ \\
Longitudinal & $\alpha_{\mathrm{LA}}$ & $2.8 \mathrm{eV}$ \\
Longitudinal & $\beta_{\mathrm{LA}}$ & $2.5 \mathrm{eV}$ \\
Effective coupling parameters & & \\
Sound velocity & $c_{\mathrm{eff}}$ & $20.0 \times 10^{3} \mathrm{~m} / \mathrm{s}$ \\
Deformation potential & $\Xi_{\mathrm{eff}}$ & $6.8 \mathrm{eV}$ \\
\hline \hline
\end{tabular}

transition matrix element is given by

$$
\begin{aligned}
P_{\mathbf{k} \mathbf{k}^{\prime}}^{\lambda}= & \frac{2 \pi}{\hbar} \sum_{\mathbf{q}}\left|g_{\mathbf{q} \lambda}\right|^{2}\left[N_{\mathbf{q} \lambda} \delta\left(\varepsilon_{\mathbf{k}^{\prime}}-\varepsilon_{\mathbf{k}}-\hbar \omega_{\mathbf{q} \lambda}\right)\right. \\
& \left.+\left(1+N_{\mathbf{q} \lambda}\right) \delta\left(\varepsilon_{\mathbf{k}^{\prime}}-\varepsilon_{\mathbf{k}}+\hbar \omega_{\mathbf{q} \lambda}\right)\right],
\end{aligned}
$$

where the two terms account for absorption and emission of phonons, respectively, $\hbar \omega_{\lambda}=\hbar c_{\lambda} q$ is the phonon energy, $c_{\lambda}$ is the acoustic sound velocity, and $g_{\mathbf{k q}}^{\lambda}$ is the electron-phonon coupling. In the following, these quantities have been obtained from first principles. The resulting values are summarized in Table I together with other parameters used in this work. The phonons are assumed to be in equilibrium and populated according to the Bose-Einstein distribution function $N_{\mathbf{q} \lambda}=$ $N\left(\hbar \omega_{\mathbf{q} \lambda}\right)$. As scattering on both the TA and LA phonon is considered here, the total relaxation time for the $K, K^{\prime}$ valleys is given by the sum of the individual phonon contributions as $\tau_{K, K^{\prime}}^{-1}=\sum_{\lambda} \tau_{\lambda}^{-1}$. As we show in the following section, the matrix elements of the electron-phonon coupling differ in the $K$ and $K^{\prime}$ valleys (see Fig. 1). The Boltzmann equation must therefore be solved explicitly in both valleys. In the absence of intervalley scattering which couples the distribution functions in the two valleys, this can be done by considering the two valleys separately. In this case, the relaxation time entering the expression for the mobility in Eq. (1) becomes the valley-averaged relaxation time $\tau=\left(\tau_{K}+\tau_{K^{\prime}}\right) / 2$, where $\tau_{K / K^{\prime}}$ is the total relaxation time in the individual valleys. Screening of the electron-phonon interaction by the carriers themselves ${ }^{42,43}$ has been considered elsewhere ${ }^{21}$ and will here be neglected.

While analytic considerations have been given in Refs. 20 and 21 , we will in the present work resort to a numerical evaluation $^{44,45}$ of the relaxation time in Eqs. (3) and (4). This allows us to study the acoustic phonon-limited mobility in graphene with the full angular dependence of the true coupling matrix elements which is more complex than most often assumed (see, e.g., Ref. 20). The numerical approach also allows for a unified treatment of the high-temperature $\left(T>T_{\mathrm{BG}}\right)$ and Bloch-Grüneisen $\left(T<T_{\mathrm{BG}}\right)$ regimes. We note, however, that in the low-temperature regime where $\hbar \omega_{\mathbf{q}} \sim$ $k_{\mathrm{B}} T$, it is crucial that the phonon energy is retained in the 
Fermi function in Eq. (3). In the high-temperature regime this requirement can be relaxed and the relaxation time can be put on a simple analytic form.

\section{INTERACTION WITH ACOUSTIC PHONONS}

In the following, we use a first-principles DFT approach to calculate the acoustic electron-phonon couplings in graphene. ${ }^{38,39,46}$ It is based on fully microscopic description of both the electronic states and the phonons. Due to their high phonon energies (>100 meV), acoustic intervalley and optical phonons do not play a role in the considered temperature range and can therefore be neglected.

The interaction between charge carriers and the acoustic phonons in graphene can be written in the general form,

$$
g_{\mathbf{k q}}^{\lambda}=\sqrt{\frac{\hbar}{2 A \rho \omega_{\mathbf{q} \lambda}}} M_{\mathbf{k q}}^{\lambda},
$$

where $A$ is the area of the graphene layer, $\rho$ is the mass density, and $M_{\mathbf{k q}}^{\lambda}=\left\langle\mathbf{k}+\mathbf{q}\left|\delta V_{\mathbf{q} \lambda}\right| \mathbf{k}\right\rangle$ is the coupling matrix element for scattering between the two Bloch states $\mathbf{k}$ and $\mathbf{k}+\mathbf{q}$ due to a phonon with wave vector $\mathbf{q}$ and branch index $\lambda$. The coupling is mediated by the change $\delta V_{\mathbf{q} \lambda}$ in the microscopic crystal potential due to a unit displacement of the atoms along the mass-scaled normal mode vector $\mathbf{e}_{\mathbf{q} \lambda}$. Due to the full microscopic treatment of both electrons and phonons, the full symmetry of both the electronic states and the phonon modes as well as umklapp processes involving reciprocal lattice vectors are included in the calculated coupling matrix elements.

Figure 1 shows the absolute value of the calculated coupling matrix elements $M_{\mathbf{k q}}^{\lambda}$ for the TA and LA phonons in the $K, K^{\prime}$ valleys as a function of the two-dimensional phonon wave vector $\mathbf{q}$. The matrix elements in the two valleys are related through time-reversal symmetry as $M_{\mathbf{k q}}^{K \lambda^{*}}=M_{-\mathbf{k},-\mathbf{q}}^{K^{\prime} \lambda}$. This implies that carriers in the two valleys traveling in the same direction experience different electron-phonon couplings, and that the Boltzmann equation must be solved explicitly in both valleys.

In order to emphasize the effect of the chirality of the carriers in graphene, the initial carrier state $\mathbf{k}$ is located on the right side of the Dirac cones $\sim 300 \mathrm{meV}$ above the $K, K^{\prime}$ points as indicated by the sketch in the top of Fig. 1. As is evident, both the TA and LA phonons couple to the carriers with similar coupling strengths. However, while backscattering is suppressed for the LA mode, the situation is reversed for the TA mode where forward scattering is suppressed. In addition to suppression of forward and backscattering, other directions with complete suppression of scattering also appear. This is a consequence of the inclusion of the symmetry of both phonons and electronic states.

In the following, the first-principles coupling matrix elements are analyzed using the group-theoretical analysis of the electron-phonon interaction presented in Ref. 40. In the long-wavelength limit, the TA and LA phonons are strictly transverse and longitudinal, respectively, ${ }^{47}$ and the electronphonon interaction has a simple analytic representation in the two-dimensional pseudospin basis. ${ }^{40}$ Using the results of Ref. 40, the coupling matrix elements can be expressed in terms of the angles $\theta_{\mathbf{k}}, \theta_{\mathbf{q}}$, and $\theta_{\mathbf{k}+\mathbf{q}}$ of the involved wave
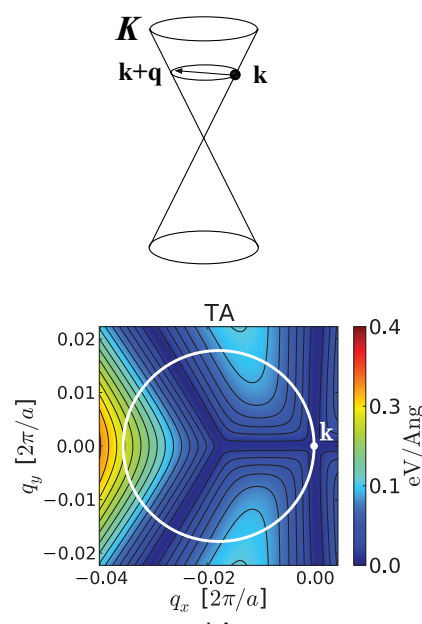$$
\text { LA }
$$
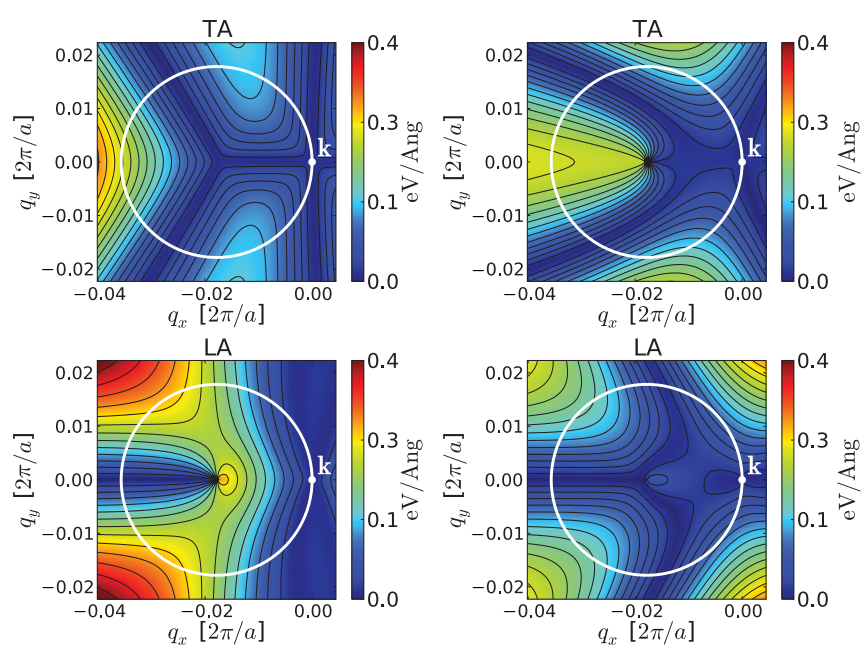

LA

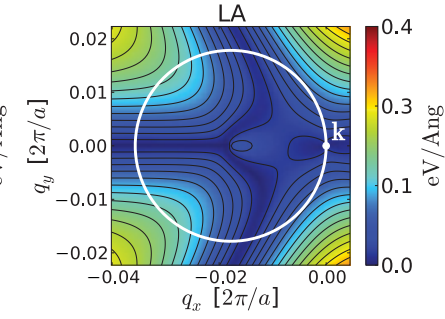

FIG. 1. (Color online) Electron-phonon couplings to the acoustic TA and LA phonons in the $K, K^{\prime}$ valleys (left and right columns, respectively) of graphene. The contour plots show the absolute value of the coupling matrix elements $\left|M_{\mathbf{k q}}^{\lambda}\right|$ for a carrier energy of $\varepsilon_{\mathbf{k}}=$ $300 \mathrm{meV}$ as a function of the two-dimensional phonon wave vector $\mathbf{q}$. The (white) circles correspond to $\mathbf{k}+\mathbf{q}$ vectors lying on the constant energy surfaces of the Dirac cones with energy $\varepsilon_{\mathbf{k}}$ as sketched in the top row.

vectors. Including contributions of order $O(q)$, we find that the coupling matrix elements in the long-wavelength limit take the following form in the $K$ valley,

$$
\left|M_{\mathbf{k q}}^{\mathrm{TA}}\right|=q \beta\left|\sin \left(2 \theta_{\mathbf{q}}+\frac{\theta_{\mathbf{k}}+\theta_{\mathbf{k}+\mathbf{q}}}{2}\right)\right|
$$

and

$$
\begin{aligned}
\left|M_{\mathbf{k q}}^{\mathrm{LA}}\right|= & q \mid \alpha \cos \left(\frac{\theta_{\mathbf{k}+\mathbf{q}}-\theta_{\mathbf{k}}}{2}\right) \\
& +\beta \cos \left(2 \theta_{\mathbf{q}}+\frac{\theta_{\mathbf{k}}+\theta_{\mathbf{k}+\mathbf{q}}}{2}\right) \mid,
\end{aligned}
$$

for the TA and LA phonons, respectively. For the LA phonon, the first and second terms originate from the deformation potential and the gauge field coupling mechanisms, respectively. The TA phonon couples only through the latter. ${ }^{40,48,49}$ Since both mechanisms are Coulombic in nature, the overall couplings in Eqs. (6) and (7) are here referred to as deformation potential couplings.

With the coupling parameters listed in Table I, we find that the analytic expressions for the electron-phonon interaction in Eqs. (6) and (7) to a high degree reproduce the first-principles matrix elements for electron energies up to $\sim 750 \mathrm{meV}$. As the analytic coupling matrix elements are based on the phonon modes in the long-wavelength limit, the agreement is slightly 
worsened at shorter wavelengths where the mode vectors deviate from the long-wavelength modes. ${ }^{47}$ This is most pronounced for the TA phonon. As the LA phonon retains its long-wavelength character far out in the Brillouin zone, the agreement between the coupling matrix elements here remains quantitative even at shorter wavelengths. In the BG regime where short-wavelength phonons are frozen out, we note that it is the long-wavelength limit of the coupling matrix elements that governs the scattering of carriers.

Often scattering on acoustic phonons is described by coupling to a single effective phonon mode with a coupling matrix element given by ${ }^{20,34}$

$$
M_{\mathrm{kq}}^{\mathrm{eff}}=\Xi_{\mathrm{eff}} q \cos \left(\frac{\theta_{\mathbf{k}, \mathbf{k}+\mathbf{q}}}{2}\right),
$$

where $\Xi_{\text {eff }}$ is the effective deformation potential and the angular part corresponds to the bare spinor overlap $\left\langle\chi_{\mathbf{k}+\mathbf{q}} \mid \chi_{\mathbf{k}}\right\rangle$ of the electronic wave function. In contrast to the more complex angular dependence of the coupling matrix element predicted by the full microscopic treatment presented here, the angular dependence of the effective coupling matrix element above suppresses only backscattering. In the high-temperature regime where equipartitioning of the acoustic phonons $N_{\mathbf{q}} \sim$ $k_{\mathrm{B}} T / \hbar \omega_{\mathbf{q}}$ applies, the relaxation time and the resistivity take the following simple forms: ${ }^{9,20}$

$$
\frac{1}{\tau_{\mathbf{k}}}=\frac{1}{\hbar^{3}} \frac{\Xi_{\mathrm{eff}}^{2} k_{\mathrm{B}} T}{4 \rho v_{F}^{2} c_{\mathrm{eff}}^{2}} \varepsilon_{\mathbf{k}}, \quad \rho=\frac{\pi \Xi_{\mathrm{eff}}^{2} k_{\mathrm{B}} T}{4 e^{2} \hbar \rho v_{F}^{2} c_{\mathrm{eff}}^{2}},
$$

where the factor of 4 in the denominators stems from the chiral nature of the carriers through the assumed form of the coupling matrix element in Eq. (8). These expressions are used almost exclusively to extract the value of the effective acoustic deformation potential in experimental situations. ${ }^{5,7-9,11}$

\section{RESULTS}

In the following, we study the intrinsic acoustic phononlimited mobility and quasiparticle lifetime of electronic carriers in graphene in both the BG and linear resistivity regime using the full coupling matrix elements as given by Eqs. (6) and (7). This allows us to establish the value of the intrinsic effective acoustic deformation potential in graphene.

\section{A. Mobility}

In Fig. 2 we show the inverse of the valley-averaged relaxation time as a function of energy for different temperatures and a carrier density of $n=10^{12} \mathrm{~cm}^{-2}$ corresponding to $\varepsilon_{F} \sim 117 \mathrm{meV}$ and $T_{\mathrm{BG}} \approx 57 \mathrm{~K}$ for the LA phonon. Above the BG temperature, the inverse relaxation time has the linear energy dependence of Eq. (9) and a slope proportional to the temperature. As the temperature is decreased below $T_{\mathrm{BG}}$, the freezing out of short-wavelength phonons and the sharpening of the Fermi surface result in limited phase space for phonon scattering and an increased lifetime of the carriers at the Fermi energy. In the expression for the relaxation time in Eq. (3), this effect is accounted for by the Fermi and Bose distribution functions. The limited phase space available for phonon scattering manifests itself in the characteristic dip at the Fermi energy that evolves in the inverse relaxation time

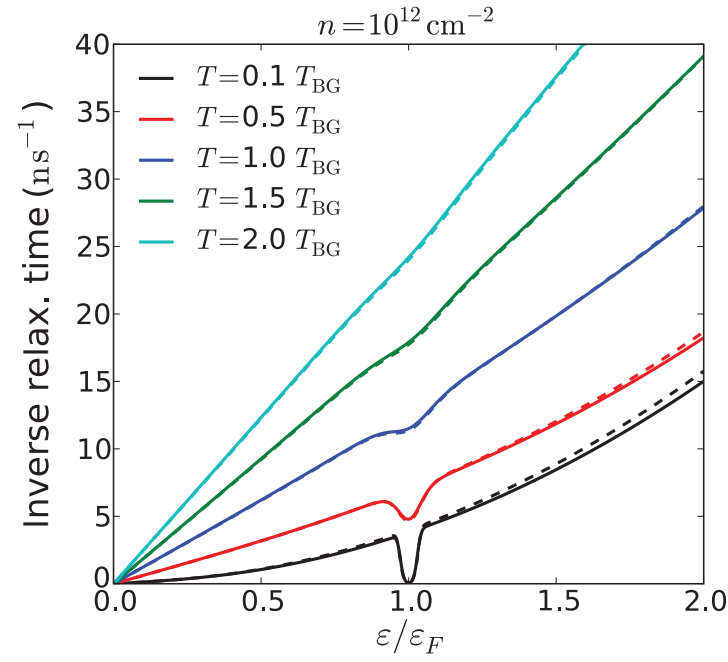

FIG. 2. (Color online) Inverse relaxation time (valley-averaged) for acoustic phonon scattering on the TA and LA phonon in the BG regime at $n=10^{12} \mathrm{~cm}^{-2}\left(T_{\mathrm{BG}} \approx 57 \mathrm{~K}\right.$ for the LA phonon). The full lines show the results obtained with the full matrix elements given in Eqs. (6) and (7) and coupling constants extracted from $a b$ initio calculations. The dashed lines show the result obtained with a single effective acoustic phonon with the coupling matrix element in Eq. (8), a deformation potential of $\Xi_{\text {eff }}=6.8 \mathrm{eV}$, and sound velocity $c_{\text {eff }}=20 \times 10^{3} \mathrm{~m} / \mathrm{s}$.

with decreasing temperature. ${ }^{20}$ For all temperatures, the linear energy dependence of the high-temperature result in Eq. (9) is recovered in the $\varepsilon \rightarrow 0$ limit.

By inspecting the individual contributions, we find that the inverse relaxation time to a large extent is dominated by the TA phonon both in the high-temperature and the BG regime. In the high-temperature regime, the domination of the TA phonon can be attributed to a number of factors. First of all, from Eq. (9) it follows directly that the lower sound velocity of the TA phonon leads to a higher scattering rate. Secondly, the coupling matrix element for the TA phonon allows for backscattering which is suppressed for the LA phonon. In the $\mathrm{BG}$ regime, the domination of the TA phonon stems from the suppression of the coupling matrix element for the LA phonon in the long-wavelength limit (see Fig. 1). Also, the lower BG temperature of the TA phonon allows for full backscattering below the BG temperature of the LA phonon. The observed dominance of the TA phonon reported here is in contrast to the often used assumption that only the LA phonon couples to charge carriers in graphene. ${ }^{20}$

In order to determine the intrinsic value of the effective deformation potential in graphene, we also calculate the relaxation time using the coupling matrix element in Eq. (8). The dashed lines in Fig. 2 show the inverse relaxation time calculated with an effective deformation potential and sound velocity of $\Xi_{\text {eff }}=6.8 \mathrm{eV}$ and $c_{\text {eff }}=20.0 \times 10^{3} \mathrm{~m} / \mathrm{s}$, respectively. It is seen to reproduce the relaxation time based on the full matrix elements very well for the energy range shown. While the extracted value for the acoustic deformation potential is much smaller than experimental values, ${ }^{5,7-9,11}$ it is in better agreement with other $a b$ initio results yielding $4.5 \mathrm{eV} .^{31}$ We also note that our findings for the value of the effective deformation potential are in stark contrast to those 

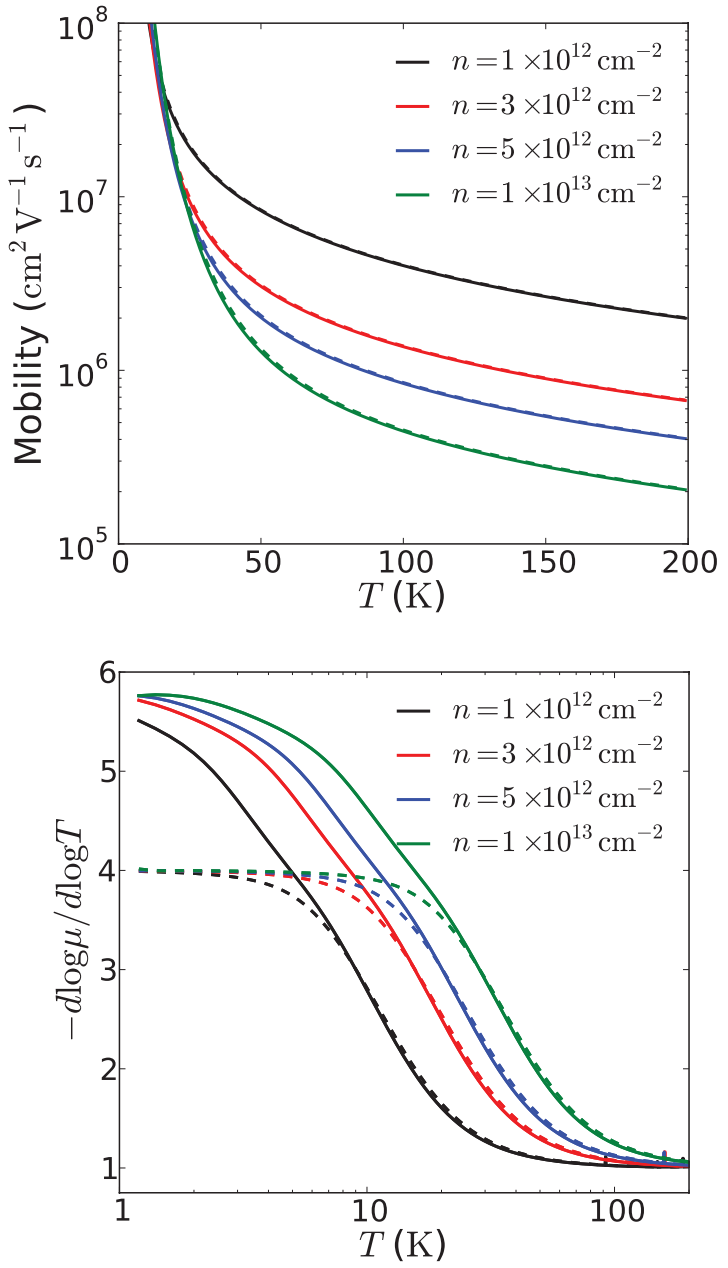

FIG. 3. (Color online) Mobility vs temperature (upper plot) for carrier densities $10^{12}-10^{13} \mathrm{~cm}^{-2}$ corresponding to $T_{\mathrm{BG}} \sim 57-180 \mathrm{~K}$ for the LA phonon. The lower plot shows the temperature dependence of the exponent $\alpha$ in the temperature dependence $\mu \sim T^{-\alpha}$ of the mobility. The dashed lines show the result obtained with coupling to a single effective acoustic phonon (see caption of Fig. 2).

of Ref. 37, where the combined coupling to the TA and LA phonons is found to yield a deformation potential of $10-20 \mathrm{eV}$.

Figure 3 summarizes the calculated acoustic phononlimited mobility as a function of temperature for carrier densities $10^{12}-10^{13} \mathrm{~cm}^{-2}$ which correspond to BG temperatures $T_{\mathrm{BG}} \sim 57-180 \mathrm{~K}$ for the LA phonon. The mobility calculated with the above-mentioned effective coupling parameters (dashed lines) reproduces the full calculation extremely well. For all carrier densities the mobility shows a transition from the linear $\mu \sim T^{-1}$ high-temperature behavior to a more pronounced $\mu \sim T^{-\alpha}$ temperature dependence with $\alpha>1$ in the $\mathrm{BG}$ regime. The decrease in the mobility with increasing carrier density stems from the linear density of states which provides more phase space for phonon scattering at larger values of the Fermi energy. At low temperatures $T \ll T_{\mathrm{BG}}$ where scattering on the full Fermi surface is frozen out, the local value of the coupling matrix element becomes important and the mobilities for the different carrier densities approach a common value.
The lower plot in Fig. 3 shows the temperature dependence of the exponent $\alpha$ for the same set of carrier densities. From this plot it is more clear that the departure away from the linear high-temperature dependence happens at $T \sim T_{\mathrm{BG}}$. Surprisingly, the exponents obtained from the mobility calculated with the full coupling matrix elements do not saturate at $\alpha=4$ as predicted by the effective coupling matrix element (dashed lines). ${ }^{20,21}$ Hence the intrinsic phonon-limited carrier mobility cannot be accounted for by an effective acoustic phonon in this temperature regime. Even in the absence of screening, the mobility of graphene should take on a temperature dependence with $\alpha>4$ at sufficiently low temperatures. With carrier screening taken into account, this behavior should be reinforced. ${ }^{21}$ Our findings for the temperature dependence of the mobility in the $\mathrm{BG}$ regime implies that experimental observations of temperature dependencies with $\alpha>4$ do not necessarily indicate that the deformation potentials in graphene are screened by the carriers.

The purely intrinsic mobilities calculated here are significantly higher than previously reported theoretical values. ${ }^{20}$ For example, for a carrier density of $n=10^{12} \mathrm{~cm}^{-2}$, a room-temperature mobility in excess of $10^{6} \mathrm{~cm}^{2} \mathrm{~V}^{-1} \mathrm{~s}^{-1}$ is here predicted. Since there is a one-to-one correspondence between the mobility and the deformation potential, this is reflected directly in the extracted deformation potential which is considerably lower than commonly used values.

\section{B. Quasiparticle lifetime}

So far, theoretical studies of the phonon-induced lifetime broadening of the electronic quasiparticles in graphene have focused on the intrinsic optical phonons of graphene and surface optical phonons of the substrate. ${ }^{16,25-27}$ However, at low temperature where thermal broadening is small, optical phonon scattering is suppressed in the vicinity of the Fermi level by Pauli blocking via the electronic occupation factors. ${ }^{25-27}$ As the range of energies where optical phonon scattering is suppressed is limited by the thresholds for absorption and emission of optical phonons with energy $\hbar \omega_{O}$ at $\varepsilon=\varepsilon_{F} \pm \hbar \omega_{O}$, this leaves a large energy window spanning up to several hundreds of meV (depending on the optical phonons in play) where acoustic phonon scattering can dominate the quasiparticle decay rate.

The lifetime $\tau_{k}$ of the quasiparticles is related to the linewidth broadening $\Gamma_{k}$ of the spectral features through the imaginary part of the (on-shell) electronic self-energy $\Sigma_{k}$ as $\tau_{k}^{-1}=-2 \operatorname{Im} \Sigma_{k} / \hbar=\Gamma_{k} / \hbar$, where $\tau_{k}^{-1}$ is the decay rate. Within the Born approximation for the self-energy, the decay rate $\tau^{-1}$ is given by the expression for the inverse relaxation time in Eq. (3) with the replacement $\left(1-\cos \theta_{\mathbf{k}, \mathbf{k}^{\prime}}\right) \rightarrow 1 .^{50}$ Using the identities in Ref. 45 , it can be recast in the more familiar form

$$
\begin{aligned}
\frac{1}{\tau_{\mathbf{k}}}= & \frac{2 \pi}{\hbar} \sum_{\mathbf{q} \lambda}\left|g_{\mathbf{q} \lambda}\right|^{2}\left[\left(N_{\mathbf{q} \lambda}+f_{\mathbf{k}+\mathbf{q}}\right) \delta\left(\varepsilon_{\mathbf{k}+\mathbf{q}}-\varepsilon_{\mathbf{k}}-\hbar \omega_{\mathbf{q} \lambda}\right)\right. \\
& \left.+\left(1+N_{\mathbf{q} \lambda}-f_{\mathbf{k}-\mathbf{q}}\right) \delta\left(\varepsilon_{\mathbf{k}+\mathbf{q}}-\varepsilon_{\mathbf{k}}+\hbar \omega_{\mathbf{q} \lambda}\right)\right],
\end{aligned}
$$

where, as in Eq. (4), the two terms account for absorption and emission of acoustic phonons. In the high-temperature regime and with the coupling given by the effective matrix element in 
Eq. (8), this reduces to

$$
\frac{1}{\tau_{\mathbf{k}}}=\frac{1}{\hbar^{3}} \frac{\Xi_{\mathrm{eff}}^{2} k_{\mathrm{B}} T}{2 \rho v_{F}^{2} c_{\mathrm{eff}}^{2}} \varepsilon_{\mathbf{k}}=2 \pi \lambda_{\mathbf{k}} k_{\mathrm{B}} T / \hbar,
$$

which differs from the corresponding expression for the inverse relaxation time in Eq. (9) by a factor of $1 / 2$ and where the connection to the often used dimensionless coupling parameter $\lambda_{\mathbf{k}}$ is given in the last equality. ${ }^{50}$ Hence, for $T>T_{\mathrm{BG}}$, the acoustic phonon-limited decay rate is proportional to both the temperature $\tau^{-1} \sim T$ and the carrier energy $\tau^{-1} \sim \varepsilon_{\mathbf{k}}$. In the $\mathrm{BG}$ regime, the energy dependence of the decay rate is similar to the one shown in Fig. 2 for the inverse relaxation time, i.e., it develops a dip at the Fermi level for $T<T_{\mathrm{BG}}$ resulting in extremely long-lived quasiparticles in the $\mathrm{BG}$ regime.

The temperature dependence of the quasiparticle decay rate $\tau_{k}^{-1}$ at $\left(k=k_{F}\right)$ and away from $\left(k=0.9 k_{F}\right)$ the Fermi energy is summarized in Fig. 4 for both the effective (dashed lines) and the full coupling matrix elements. Interestingly, we here find that the effective deformation potential $\Xi_{\text {eff }}=5.3 \mathrm{eV}$ (corresponding to $\lambda_{k_{F}}=0.3,1.0 \times 10^{-3}$ for $n=10^{12}, 10^{13} \mathrm{~cm}^{-2}$, respectively) obtained by fits to the full decay rate, differs from the effective deformation potential extracted from the relaxation time and mobility in Figs. 2 and 3. This difference stems from the fact that the angular dependence of the matrix elements in Eqs. (6) and (7) is weighted differently in the inverse momentum relaxation time and the decay rate due to the appearance of the factor $\left(1-\cos \theta_{\mathbf{k}, \mathbf{k}^{\prime}}\right)$ in Eq. (3) for the former. Care must therefore be taken when comparing deformation potentials extracted from transport and photoemission experiments.

For temperatures $T>T_{\mathrm{BG}}$, the temperature dependence of the decay rate in Fig. 4 shows the linear $T$ dependence in Eq. (11) where the offset between the different curves originates from the density dependence of the Fermi level.

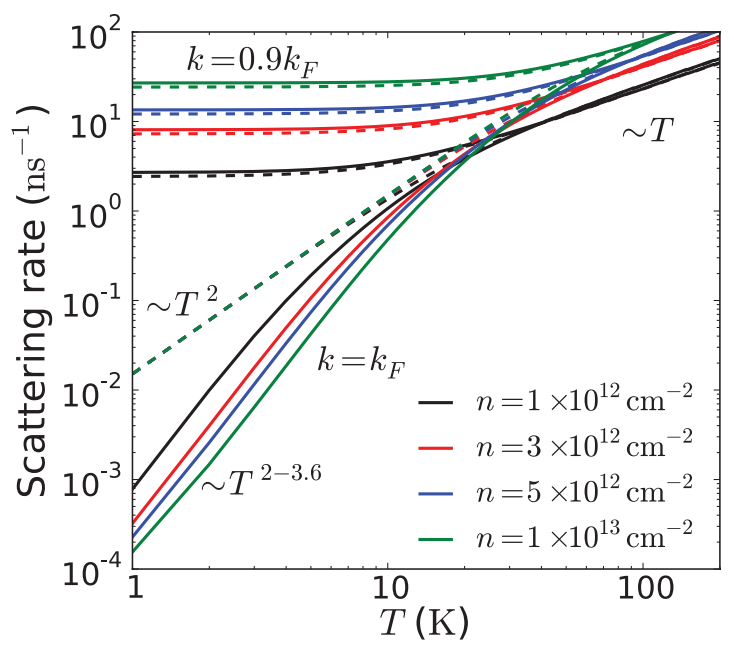

FIG. 4. (Color online) Temperature dependence of the quasiparticle decay rate $\tau_{k}^{-1}$ at the Fermi level $k=k_{F}$ and $k=0.9 k_{F}$ for acoustic phonon scattering on the TA and LA phonons. The dashed lines show the result obtained with the effective coupling matrix element in Eq. (8), a deformation potential of $\Xi_{\text {eff }}=5.3 \mathrm{eV}$, and sound velocity $c_{\text {eff }}=20 \times 10^{3} \mathrm{~m} / \mathrm{s}$.
At $T<T_{\mathrm{BG}}$, the decay rate at the Fermi level acquires a stronger temperature dependence $\tau^{-1} \sim T^{\alpha}$. As was the case for the mobility, the effective and full phonon couplings give quantitatively different results in the BG regime. First of all, the different curves for the effective coupling all collapse into one curve for $T<T_{\mathrm{BG}}$, indicating that the decay rate at the Fermi energy is independent of the density. For the full coupling, this is not the case. Secondly, while the temperature dependence for the effective coupling saturates at $\alpha=2$ for $T<T_{\mathrm{BG}}$, the exponent for the full coupling does not saturate and increases up to a value of $\alpha \sim 3$.6. Similar observations hold for the inverse relaxation time where the exponent saturates at $\alpha=4$ and $\alpha \sim 5.7$, respectively. This is the origin of the discrepancy in the temperature dependence of the mobilities shown in Fig. 3. Away from the Fermi level $\left(k=0.9 k_{F}\right)$, the effective and full couplings agree and the quasiparticle decay rate approaches a density-dependent constant value in the $\mathrm{BG}$ regime, i.e., $0<\alpha<1$. At even lower carrier energies $k \ll k_{F}$ (not shown), the high-temperature behavior in Eq. (11) is recovered for all carrier densities. We note that a similar temperature dependence of the acoustic phonon-limited decay rate has been predicted for chiral carriers in topological insulator thin films. ${ }^{51}$

Since the quasiparticle decay rates in Fig. 4 correspond to very small linewidth broadenings $\Gamma<0.1 \mathrm{meV}$, the reported linewidth signatures from acoustic phonon scattering can be hard to identify in practice where the energy resolution in photoemission spectroscopy is an order or two magnitudes higher. Furthermore, competing inelastic scattering due to electron-electron interactions also contributes to the decay rate. $^{52}$ To which extent this dominates acoustic phonon scattering depends on the temperature, the carrier density, and the value of the effective deformation potential in graphene which experimentally seems to be a factor of $\sim 3-5$ larger than the theoretical value determined here.

As a final remark, the high-temperature scattering rate at the Fermi level of $\tau^{-1} \sim 10^{11} \mathrm{~s}^{-1}$ reported in Fig. 4 corresponds to a mean-free path of $\lambda=v_{F} / \tau \sim 1000 \mathrm{~nm}$. Such an extremely large mean-free path may open the opportunity to study coherent transport in relatively large graphene structures.

\section{CONCLUSIONS AND DISCUSSIONS}

In the present study the acoustic electron-phonon interaction in graphene has been analyzed in detail. The exact analytic forms of the coupling matrix elements in the long-wavelength limit were found to match the calculated first-principles matrix elements almost quantitatively even at shorter wavelengths. As previously predicted, ${ }^{20,21}$ the mobility shows a transition from a $\mu \sim T^{-1}$ to a $\mu \sim T^{-\alpha}$ temperature dependence with $\alpha>1$ below the BG temperature. However, contrary to earlier studies we found that the full coupling matrix elements cause the temperature dependence of the mobility to increase beyond a $\alpha=4$ dependence which is otherwise only expected when screening is included. ${ }^{21}$ A similar BG transition was shown to occur in the quasiparticle decay rate which goes from a $\tau^{-1} \sim T$ temperature dependence at $T>T_{\mathrm{BG}}$ to a $\tau^{-1} \sim T^{\alpha}$ dependence with $\alpha \sim 2-3.6(0<\alpha<1)$ at (away from) the Fermi level for $T<T_{\mathrm{BG}}$. 
By replacing the coupling to the TA and LA phonons with a coupling to an effective acoustic phonon, the intrinsic acoustic deformation potential extracted from fits to the mobility (decay rate) was found to be $\Xi_{\text {eff }}=6.8 \mathrm{eV}$ (5.3 $\mathrm{eV})$. For the mobility it was shown that the effective acoustic phonon reproduces the temperature dependence correctly in the high-temperature regime, while quantitatively different results were obtained in the BG regime. This discrepancy was shown to originate in different temperature dependencies of the decay rate/inverse relaxation time at the Fermi level.

Since the inferred acoustic deformation potential is much lower than the experimentally determined values $\left(\Xi_{\text {eff }} \sim 18\right.$ $29 \mathrm{eV}$ ), our results suggest that the acoustic phonon-limited transport in substrate-supported graphene is at present not fully understood. Possible explanations for the large experimental deformation potentials could be (i) substrate-induced modifications of the band structure ${ }^{53,54}$ that modifies the chirality of the electronic states (and thereby the angular dependence of the coupling matrix element) or the Fermi velocity, and (ii) the existence of additional acoustic phonons not considered in the present work as, for example, surface-acoustic phonons on the substrate which have previously been studied in 2DEGs. ${ }^{55}$ The relatively large variations in the experimental deformation potentials indeed indicate that the acoustic deformation potential is highly dependent on experimental factors such as the substrate.

\section{ACKNOWLEDGMENTS}

The authors would like to thank T. Markussen and A.-P. Jauho for useful comments on the manuscript. K.K. has been partially supported by the Center on Nanostructuring for Efficient Energy Conversion (CNEEC) at Stanford University, an Energy Frontier Research Center funded by the US Department of Energy, Office of Science, Office of Basic Energy Sciences under Grant No. DE-SC0001060. CAMD is supported by the Lundbeck Foundation. *cosby@fys.ku.dk

${ }^{1}$ K. S. Novoselov, A. K. Geim, S. V. Morozov, D. Jiang, Y. Zhang, S. V. Dubonos, I. V. Grigorieva, and A. A. Firsov, Science 306, 666 (2004).

${ }^{2}$ A. K. Geim and K. S. Novoselov, Nat. Mater. 6, 183 (2007).

${ }^{3}$ A. H. C. Neto, F. Guinea, N. M. R. Peres, K. S. Novoselov, and A. K. Geim, Rev. Mod. Phys. 81, 109 (2009).

${ }^{4}$ S. Das Sarma, S. Adam, E. H. Hwang, and E. Rossi, Rev. Mod. Phys. 83, 407 (2011).

${ }^{5}$ J.-H. Chen, C. Jang, S. Xiao, M. Ishigami, and M. S. Fuhrer, Nature Nano. 3, 206 (2008).

${ }^{6}$ E. H. Hwang and S. Das Sarma, Phys. Rev. B 79, 165404 (2009).

${ }^{7}$ K. Zou, X. Hong, D. Keefer, and J. Zhu, Phys. Rev. Lett. 105, 126601 (2010).

${ }^{8}$ C. R. Dean, A. F. Young, I. Meric, C. Lee, L. Wang, S. Sorgenfrei, K. Watanabe, T. Taniguchi, P. Kim, K. L. Shepard et al., Nature Nano. 5, 722 (2010).

${ }^{9}$ D. K. Efetov and P. Kim, Phys. Rev. Lett. 105, 256805 (2010).

${ }^{10}$ A. Pachoud, M. Jaiswal, P. K. Ang, K. P. Loh, and B. Özyilmaz, Europhys. Lett. 92, 27001 (2010).

${ }^{11}$ K. I. Bolotin, K. J. Sikes, J. Hone, H. L. Stormer, and P. Kim, Phys. Rev. Lett. 101, 096802 (2008).

${ }^{12}$ J. Xue, J. Sanchez-Yamagishi, D. Bulmash, P. Jacquod, A. Deshpande, K. Watanabe, T. Taniguchi, P. Jarillo-Herrero, and B. J. LeRoy, Nat. Mater. 10, 282 (2011).

${ }^{13}$ R. Decker, Y. Wang, V. W. Brar, W. Regan, H.-Z. Tsai, Q. Wu, W. Gannett, A. Zettl, and M. F. Crommie, Nano Lett. 11, 2291 (2011).

${ }^{14}$ C. R. Dean, A. F. Young, P. Cadden-Zimansky, L. Wang, H. Ren, K. Watanabe, T. Taniguchi, P. Kim, J. Hone, and K. L. Shepard, Nature Phys. 7, 693 (2011).

${ }^{15}$ P. J. Zomer, S. P. Dash, N. Tombros, and B. J. van Wees, Appl. Phys. Lett. 99, 232104 (2011).

${ }^{16}$ S. Fratini and F. Guinea, Phys. Rev. B 77, 195415 (2008).

${ }^{17}$ A. Konar, T. Fang, and D. Jena, Phys. Rev. B 82, 115452 (2010).

${ }^{18}$ X. Li, E. A. Barry, J. M. Zavada, M. B. Nardelli, and K. W. Kim, Appl. Phys. Lett. 97, 232105 (2010).
${ }^{19}$ J. M. Ziman, Electrons and Phonons (Oxford University Press, London, 1960).

${ }^{20}$ E. H. Hwang and S. Das Sarma, Phys. Rev. B 77, 115449 (2008).

${ }^{21}$ H. Min, E. H. Hwang, and S. Das Sarma, Phys. Rev. B 83, 161404 (2011).

${ }^{22}$ A. Bostwick, T. Ohta, T. Seyller, K. Horn, and E. Rotenberg, Nature Phys. 3, 36 (2007)

${ }^{23}$ M. Bianchi, E. D. L. Rienks, S. Lizzit, A. Baraldi, R. Balog, L. Hornekær, and P. Hofmann, Phys. Rev. B 81, 041403 (2010).

${ }^{24}$ S. Ulstrup, M. Bianchi, R. Hatch, D. Guan, A. Baraldi, D. Alfè, L. Hornekær, and P. Hofmann, e-print arXiv:1203.2187.

${ }^{25}$ C.-H. Park, F. Giustino, M. L. Cohen, and S. G. Louie, Phys. Rev. Lett. 99, 086804 (2007).

${ }^{26}$ M. Calandra and F. Mauri, Phys. Rev. B 76, 205411 (2007).

${ }^{27}$ W.-K. Tse and S. Das Sarma, Phys. Rev. Lett. 99, 236802 (2007).

${ }^{28}$ E. H. Hwang and S. Das Sarma, Phys. Rev. B 77, 081412 (2008).

${ }^{29}$ C.-H. Park, F. Giustino, C. D. Spataru, M. L. Cohen, and S. G. Louie, Phys. Rev. Lett. 102, 076803 (2009).

${ }^{30}$ L. M. Woods and G. D. Mahan, Phys. Rev. B 61, 10651 (2000).

${ }^{31}$ K. M. Borysenko, J. T. Mullen, E. A. Barry, S. Paul, Y. G. Semenov, J. M. Zavada, M. B. Nardelli, and K. W. Kim, Phys. Rev. B 81, 121412 (2010)

${ }^{32}$ V. Perebeinos and P. Avouris, Phys. Rev. B 81, 195442 (2010).

${ }^{33}$ L. Pietronero, S. Strässler, H. R. Zeller, and M. J. Rice, Phys. Rev. B 22, 904 (1980).

${ }^{34}$ T. Stauber, N. M. R. Peres, and F. Guinea, Phys. Rev. B 76, 205423 (2007).

${ }^{35}$ E. V. Castro, H. Ochoa, M. I. Katsnelson, R. V. Gorbachev, D. C. Elias, K. S. Novoselov, A. K. Geim, and F. Guinea, Phys. Rev. Lett. 105, 266601 (2010).

${ }^{36}$ R. S. Shishir and D. K. Ferry, J. Phys.: Condens. Matter 21, 232204 (2009). 
${ }^{37}$ H. Ochoa, E. V. Castro, M. I. Katsnelson, and F. Guinea, Phys. Rev. B 83, 235416 (2011).

${ }^{38}$ K. Kaasbjerg, K. S. Thygesen, and K. W. Jacobsen, Phys. Rev. B 85, 115317 (2012).

${ }^{39}$ J. Enkovaara, C. Rostgaard, J. J. Mortensen, J. Chen, M. Dulak, L. Ferrighi, J. Gavnholt, C. Glinsvad, V. Haikola, H. A. Hansen et al., J. Phys.: Condens. Matter 22, 253202 (2010).

${ }^{40}$ J. L. Mañes, Phys. Rev. B 76, 045430 (2007).

${ }^{41}$ E. H. Hwang, S. Adam, and S. Das Sarma, Phys. Rev. Lett. 98, 186806 (2007).

${ }^{42}$ B. Wunsch, T. Stauber, F. Sols, and F. Guinea, New J. Phys. 8, 318 (2006).

${ }^{43}$ E. H. Hwang and S. Das Sarma, Phys. Rev. B 75, 205418 (2007).

${ }^{44}$ Since $c_{\lambda} / v_{F} \sim 10^{-2}$ for graphene, the acoustic phonon energy can to a good approximation be neglected in argument of energy conserving $\delta$ functions of Eq. (4).

${ }^{45}$ For the numerical evaluation of Eq. (3), the identity

$$
f_{\mathbf{k}}\left(1-f_{\mathbf{k} \pm \mathbf{q}}\right) N_{ \pm \mathbf{q}}=f_{\mathbf{k} \pm \mathbf{q}}\left(1-f_{\mathbf{k}}\right)\left(1+N_{ \pm \mathbf{q}}\right),
$$

where $f_{\mathbf{k} \pm \mathbf{q}}=f\left(\varepsilon_{\mathbf{k}} \pm \hbar \omega_{\mathbf{q}}\right)$ and $N_{ \pm \mathbf{q}}=N\left( \pm \hbar \omega_{\mathbf{q}}\right)$ is understood, comes in very handy.
${ }^{46}$ The coupling matrix elements for the electron-phonon coupling have been calculated within DFT-LDA using a $7 \times 7$ supercell and a double-zeta polarized (DZP) basis for the electronic Bloch states.

${ }^{47}$ At shorter wavelengths, the strictly transverse and longitudinal character of the modes vanishes. For the TA phonon the transverse character is only retained in certain high symmetry directions of the Brillouin zone. For the LA phonon we find that the mode vector retains its long-wavelength character for $q$ values up to $\sim 30 \%$ of the Brillouin zone size.

${ }^{48}$ E. Mariani and F. von Oppen, Phys. Rev. Lett. 100, 076801 (2008).

${ }^{49}$ E. Mariani and F. von Oppen, Phys. Rev. B 82, 195403 (2010).

${ }^{50} \mathrm{G}$. Grimvall, The Electron-Phonon Coupling in Metals (NorthHolland, Amsterdam, 1981).

${ }^{51}$ S. Giraud, A. Kundu, and R. Egger, Phys. Rev. B 85, 035441 (2012).

${ }^{52}$ E. H. Hwang, B. Y.-K. Hu, and S. Das Sarma, Phys. Rev. B 76, 115434 (2007).

${ }^{53}$ C.-H. Park, L. Yang, Y.-W. Son, M. L. Cohen, and S. G. Louie, Phys. Rev. Lett. 101, 126804 (2008).

${ }^{54}$ C. Ortix, L. Yang, and J. van den Brink, e-print arXiv:1111.0399.

${ }^{55}$ A. Knäbchen, Phys. Rev. B 55, 6701 (1997). 